\title{
Effect of Electrical Stimulation on Middle Latency Response in the Guinea Pig
}

\author{
Stephen C. Cannon, MD, Josef M. Miller, PhD, John Crowther, MD, FRCS, and Dan Moscow, MS
}

\begin{abstract}
A temporary threshold shift (TTS) has been demonstrated in the electrically evoked middle latency response (EMLR) following exposure to moderate levels of continuous electrical stimulation via a cochlear implant. The threshold at which the EMLR was elicited in chronically implanted guinea pigs was elevated by approximately $100 \%$ following 30 minutes of moderate intensity (200 $\mu \mathrm{A}$ or more) sinusoidal electrical stimulation of the cochlea. Results obtained under anesthesia varied unacceptably. In awake animals, EMLR thresholds were stable over time and consistant TTSs were observed. The threshold returned to prestimulation levels within 4 hours following termination of the stimulation. The possibility of histopathologic changes and the relevance of these findings in setting safe output levels for cochlear implant processors are discussed. AM J OTOLARYNGOL 11:251-255. (c) 1990 by W.B. Saunders Company.

Key words: cochlear prosthesis, middle latency response, guinea pig, temporary threshold shift, high intensity cochlear stimulation.
\end{abstract}

Due to the importance of defining and reducing the risks associated with cochlear implantation and stimulation in humans, considerable research has been directed toward developing measures to detect harmful changes. Following damage to the eighth nerve in animals, changes in the evoked responsiveness of the auditory system have been reported after acute and chronic electrical stimulation. ${ }^{1-4}$ In these studies, temporary and permanent threshold shifts in the electrically evoked acoustic brainstem response (EABR) have been used as a means of measuring results from intense electrical or acoustic stimulation of the cochlea. This measurement has also been related to cochlear pathology in pharmacologically damaged guinea pig cochleae ${ }^{5,6}$ and in cat cochleae damaged by either pharmacologic or mechanical trauma. ${ }^{1,7,8}$

Despite the potential utility of the EABR to evaluate the auditory system, there are some drawbacks inherent in its use. In studies of the cochlear prosthesis, its sensitivity to electrical artifact is a

Received August 7, 1989 from the University of Michigan, Kresge Hearing Research Institute, Ann Arbor, MI. Accepted for publication October 10, 1989.

Supported in part by NIH grants no. NS 21440 and NS 07293 and FDA contract 223-87-6028.

Address correspondence and reprint requests to Josef $\mathrm{M}$. Miller, PhD, Kresge Hearing Research Institute, The University of Michigan, Ann Arbor, MI 48109-0506.

(C) 1990 by W.B. Saunders Company.

0196-0709/90/1104-0005\$5.00/0 major disadvantage. Because it includes short latency components of the auditory system's evoked response (a desirable feature for studies of the auditory periphery, including the eighth nerve and brainstem), it is also affected by the artifacts associated with electrical stimulation. In acute animal studies in which time is available for the manipulation of reference and grounding electrodes, this problem can usually be overcome. Chronic preparations and humans present greater difficulties; howcver, with special carc, the EABR can be used as a reliable indicator of system responsiveness. ${ }^{9,10}$

The middle latency response (MLR) may provide an alternative measure. Its longer latency waveforms are less susceptible to electrical stimulus artifact and permit the use of longer, more effective pulse durations than are possible with the EABR. ${ }^{11}$ Moreover, the acoustic MLR has been demonstrated to be more closely related to behavioral thresholds than is the ABR, ${ }^{12}$ and the threshold for an electrically evoked middle latency response (EMLR) has been shown to match closely electrically elicited behavioral thresholds. ${ }^{10,11}$

The MLR has been described in humans as well as in several animal models. ${ }^{11,13}$ In the guinea pig, it is a triphasic response that occurs from $\mathbf{1 0}$ to $\mathbf{5 0}$ msec following acoustic or electric stimulation of the cochlea. ${ }^{14-17}$ The generator site appears to be the temporal lobes, bilaterally, in humans ${ }^{18,19}$ and the contralateral auditory cortex in guinea pigs. ${ }^{15}$ Dogs and monkeys show a hemispheric distribu- 
tion lying between that of guinea pigs and humans, with the monkey more similar to humans and the dog MLR more similar to the guinea pig. ${ }^{13}$ Like the EABR, the EMLR has been related to eighth nerve degeneration. Jyung et $\mathrm{al}^{20}$ found the slope of the EMLR input/output $(\mathrm{I} / \mathrm{O})$ function to correspond to the number of surviving spiral ganglion cells; however, spiral ganglion cell loss was not associated with a threshold change in the EMLR.

In this experiment, the effects of short-term, moderately intense electrical cochlear stimulation on the EMLR of guinea pigs were evaluated. We hypothesized that, like the EABR, this measurement would show a temporary threshold shift (TTS) ${ }^{3}$ that would recover over time to prestimulation values. The threshold for obtaining the MLR was measured, as was the amplitude and latency of MLR wave components after continuous sinusoidal electric stimulation at various levels.

\section{MATERIALS AND METHODS}

Pigmented guinea pigs with intact hair cells, weighing 250 to $400 \mathrm{~g}$ each, underwent aseptic surgery under ketamine and xylazine general anesthesia. Two epidural stainless steel recording screws were self-tapped into holes drilled in the cranium. The active electrode was placed over the auditory cortex $1 \mathrm{~cm}$ lateral to the bregma, while a reference electrode was placed $2 \mathrm{~cm}$ anterior to the bregma. ${ }^{14.15}$ The round window contralateral to the implanted auditory cortex was then exposed by opening the mastoid bulla through a postauricular incision and a platinum-iridium ball electrode, $0.25 \mathrm{~mm}$ in diameter, was positioned onto the round window membrane. A reference electrode was placed through a hole drilled in the adjacent bulla wall, and a 5 -mm noninsulated portion placed well in contact with the mucosa lining the bulla. Both of these electrodes, fastened to a two-pin socket, were secured to the vertex with self-tapping stainless steel screws and dental acrylic. An additional screw, to be used as a stabilizing device during recording sessions, was inverted and mounted on the skull with dental acrylic.

Sixteen animals were used in acute experiments, primarily to determine the optimal position of recording electrodes and the parameters of stimulation producing a threshold shift. These studies were performed under general anesthesia. An additional eight animals were used for recording unanesthetized responses in chronic preparations. Stimuli were $400 \mu \mathrm{sec}$ biphasic (200 $\mu$ sec/phase) charge-balanced square waves, presented at a rate of $5 / \mathrm{sec}$ by an isolated constant current stimulator. A needle in the neck muscle served as a ground electrode. Recorded signals were amplified 10,000 times by a Grass P15 preamplifier with filters at $3 \mathrm{~Hz}$ to $3 \mathrm{kHz}$ and a second stage broadband amplifier (constructed in-house). Two hundred samples of $80 \mathrm{msec}$ of neural response following stimulation were averaged using an $\mathrm{MI}^{2}$ (Modular Instruments, Southeastern, PA) signal processor. In these subjects, threshold determinations were made and $I / O$ functions were obtained. Threshold measures were based on visual detection. The intensity of electrical stimuli was presented in a counter-balanced fashion from threshold up to $800 \mu \mathrm{A}$ to obtain the I/O functions. From these values, amplitude-intensity and latency-intensity functions were derived. Amplitude measurements were made by evaluating peak-to-peak response between waves $A-B$ and B-C. Latency measurements were made from stimulus onset to the peaks of waves $A, B$, and $C$.

In the acute study, threshold and $\mathrm{V} O \mathrm{O}$ functions were measured immediately following implantation. Following these baseline measurements, a $1,000-\mathrm{Hz}$ constant sinusoidal current of 200,250 , or $300 \mu \mathrm{A}$ (RMS) was delivered to the cochlea for 30 minutes. Repeat threshold and IOO functions were then measured at 2, 10, and 30 minutes and at 1,2, and 4 hours after stimulation. A measurement was taken 24 hours after stimulation if complete recovery was not seen by 4 hours poststimulation. The chronic animal protocol varied from the protocol for acute animals in that, after surgical implantation, the animal was allowed to recover for at least 24 hours before initial measurements were made. These and all subsequent measurements in the chronic animals were taken with the animal awake and gently restrained.

\section{RESULTS}

Acute Study. The acute experiments demonstrated optimal recording sites over the contralateral auditory cortex. They also displayed significant changes in sensitivity of the response with small changes in recording sites. These findings for electrical stimulation were similar to the observations of Burton et $\mathrm{al}^{16,17}$ and Kraus et $\mathrm{al}^{15}$ for acoustical stimulation. In these acute preparations, moderately intense stimulation for $30 \mathrm{~min}$ utes was found to elevate the threshold of the EMLR in a reversible manner, thus producing a TTS. The threshold for EMLR-TTS was three to five times above the prestimulation threshold for eliciting an EMLR. However, in these studies, both the EMLR and EMLR-TTS effect were quite variable, largely due to the use of general anesthesia. Both amplitude and latency were affected by the anesthesia. The general pattern consisted of an initial amplitude depression followed by an increased slope of the I/O function as the animal recovered from anesthesia. These effects of anesthesia on the EMLR have been reported by Crowther et al. ${ }^{21}$ While the EMLR threshold remained relatively stable during anesthesia, the marked changes in response amplitude made reliable threshold measurement difficult and variable. Concern over the variability in this preparation led to the development of the chronic preparation described above.

Chronic Study. The observed EMLRs for different intensities of stimulation is shown in Fig 1. In the individual chronic animal, the EMLR threshold was consistent over time, as was the amplitude and latency of the waveform. Thresholds varied from 30 to $100 \mu \mathrm{A}$ across animals, presumably based on the positioning of recording 


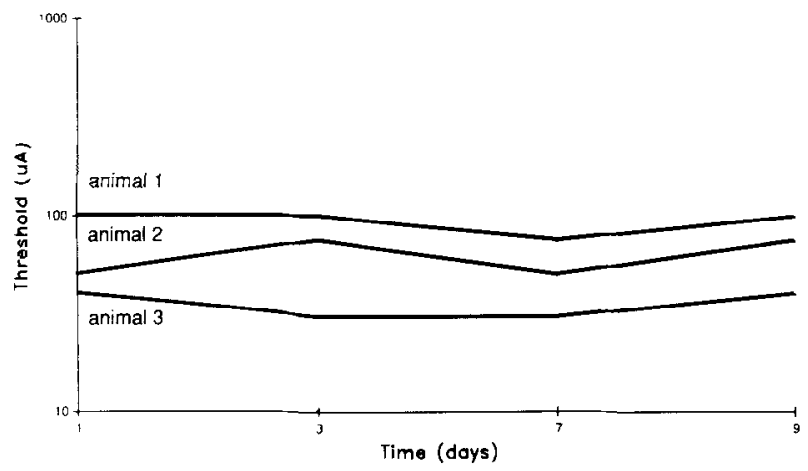

Figure 1. Evoked middle latency responses from an optimal recording site at threshold $(75 \mu \mathrm{A})$ and suprathreshold values.

and stimulating electrodes. Animals followed over several hours showed stable threshold, amplitude, and latency functions. Specifically, measurements made thruughout the period required for the experimental stimulation session demonstrated that EMLRs remained stable in the absence of stimulation and that responses measured in the same animals at daily or weekly intervals were consistent. Figure 2 illustrates threshold observation.

Continuous sinusoidal stimulation for $30 \mathrm{~min}-$ utes at each of the levels tested $(200,250$, and 300 4 A) yielded an elevation in the EMLR threshold. With all levels of stimulation, however, thresholds recovered to prestimulation levels by 4 hours. Figures 3 through 5 demonstrate the mean EMLR thresholds before and up to 2 hours after stimulation. As can be seen in each group, there was a significant threshold shift with recovery to near prestimulation level by the end of 2 hours. The mean percent of threshold shift immediately poststimulation (Fig 6) was $188 \%$ of the initial value after $200 \mu \mathrm{A}, 204 \%$ after $250 \mu \mathrm{A}$, and $223 \%$ of the initial threshold after $300 \mu \mathrm{A}$ sinusoidal stimulation. While a trend relating stimulus intensity to the amount of threshold shift and recovery was observed, a regression analysis revealed an insignificant correlation between the stimulus

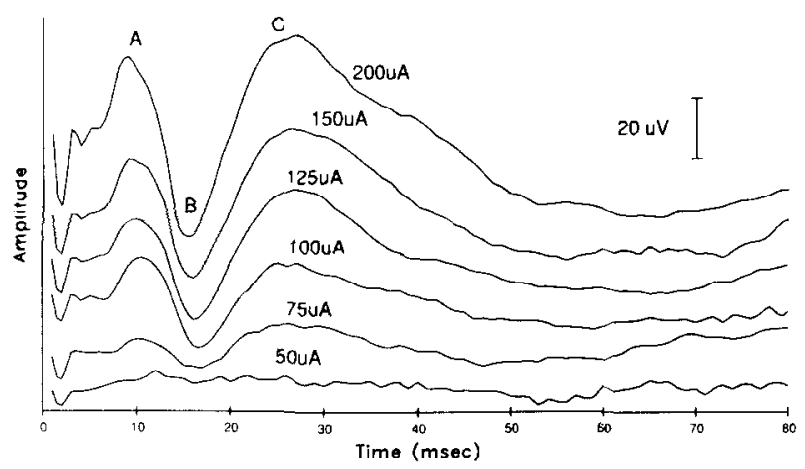

Figure 2. Evoked middle latency response thresholds show consistency over time in three animals without sinusoidal electrical stimulation

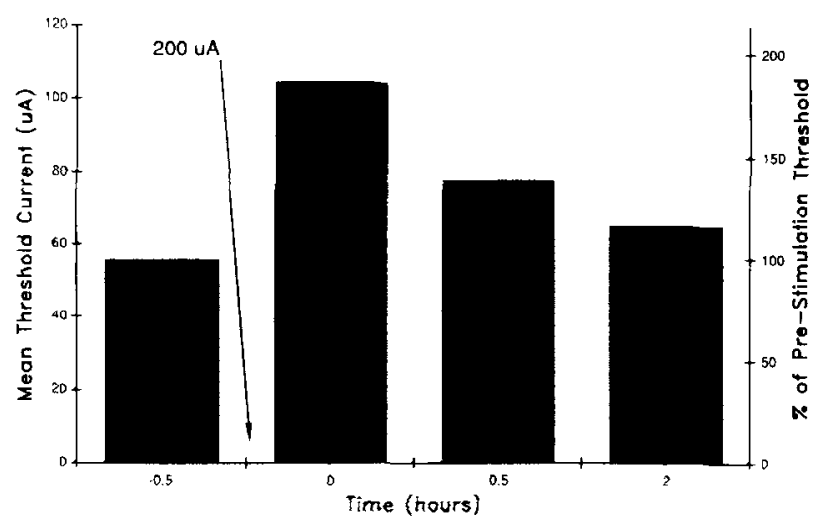

Figure 3. Mean threshold values (23 observations in five animals) before and after 30 minutes of $200 \mu \mathrm{A}$ sinusoidal electrical stimulation.

level and the degree of initial threshold shift; in addition, no significant relationship was observed between the rate or degree of recovery and the level of sinusoidal stimulation.

Examples of I/O functions generated before and after 200 and $300 \mu \mathrm{A}$ stimulation, based on the A-B peak-to-peak amplitude, are shown in Fig 7. A significant initial depression of amplitude is followed by recovery to near prestimulation levels by 2 hours. Of the eight animals studied, two showed no significant change in amplitude and, in one, a significant artifact prevented a meaningful measurement of amplitudes. The variability across animals for I/O amplitude functions was significant. In contrast, functions were stable in control trials in which repeat I/O measurements were made without stimulation, demonstrating no significant change in A-B amplitude or latency.

\section{DISCUSSION}

This study described methods for measuring chronic EMLRs in the guinea pig. A restraining bolt fixed to the head of the animal and the use of a specially designed restraining device permitted prolonged recording of reproducible EMLRs in

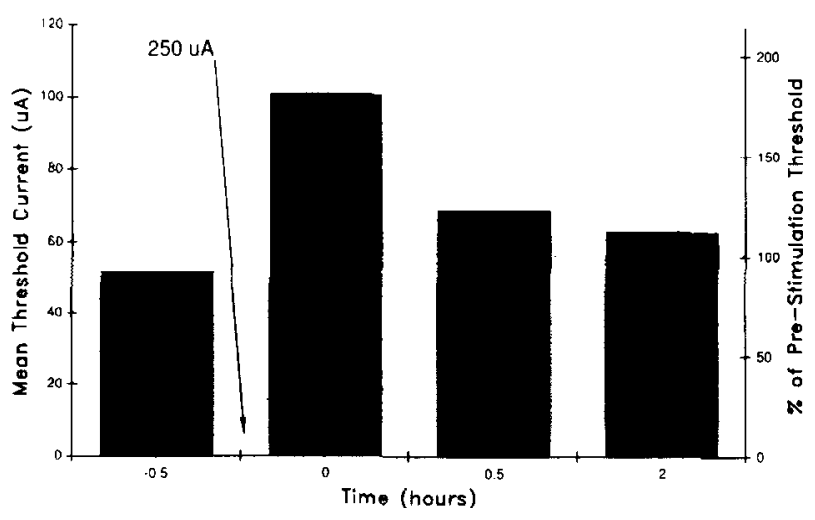

Figure 4. Mean threshold values (12 observations in three animals) before and after 30 minutes of $250 \mu \mathrm{A}$ sinusoidal stimulation. 


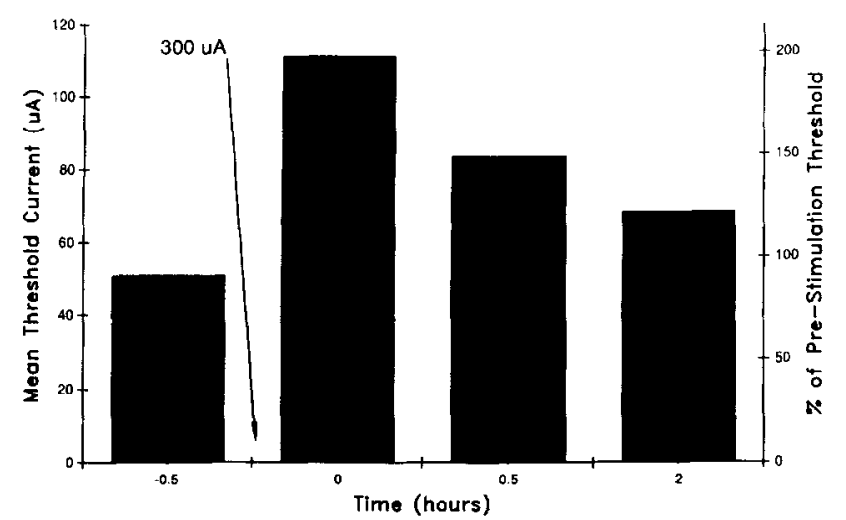

Figure 5. Mean threshold values (19 observations in three animals) before and after 30 minutes of $300 \mu \mathrm{A}$ sinusoidal stimulation.

the awake guinea pig with little apparent discomfort to the animal. It also demonstrated that a TTS occurs in the threshold of the EMLR following muderately high-intensity stimulation. Analysis of the EMLR-TTS was possible only in the chronic preparation because, in the acute preparation, there was variability in response characteristics caused by general anesthesia. However, the basic effect was clear in both preparations.

The TTS we observed in the EMLR of the guinea pig after $1,000 \mathrm{~Hz}$ sinusoidal electric stimulation of the scala tympani at current levels of 200 to 300 $\mu \mathrm{A}$ is similar to that demonstrated in an earlier study in the EABR of the guinea pig following electrical stimulation. ${ }^{3}$ In that study, sinusoidal stimulation at 100,200 , or $300 \mu \mathrm{A}$ was delivered repeatedly to the promontory, the round window, or the scala tympani. Threshold elevations recovered to baseline threshold levels following each stimulus. A temporary increase in the latency of the EABR was associated with a depression of EABR amplitude. A variation in the percent change in threshold according to site of stimulation was observed with the greatest change noted at the scala tympani and the least change noted with promontory stimulation.

The basic phenomenon of EABR-TTS was rep-

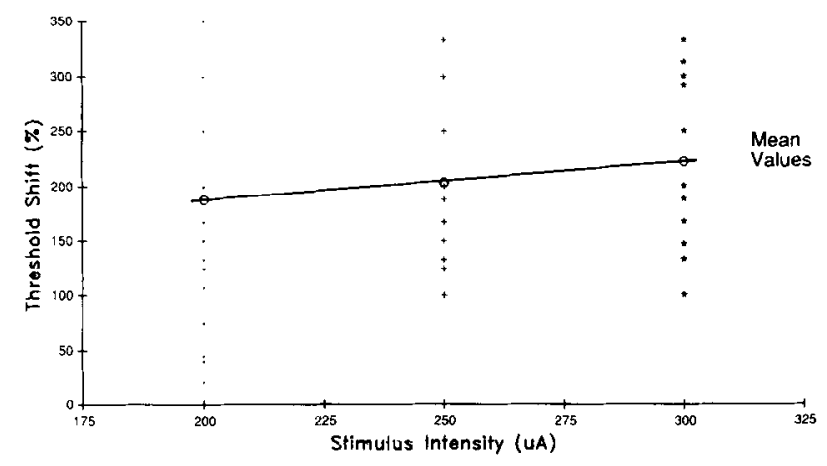

Figure 6. Mean temporary threshold shifts of $188 \%$ after 200 $\mu A(N=23), 204 \%$ after $250 \mu A(N=12)$, and $223 \%$ after 300 $\mu \mathrm{A}(\mathrm{N}=19)$ sinusoidal stimulation are seen. licated in our EMLR study. We also noted a statistically insignificant trend linking increased slope of the I/O amplitude function with the EMLR-TTS. Initial values were recorded 2 minutes after the cessation of stimulation in accordance with the convention of typical acoustic TTS studies. Theoretically, this would bypass any early artifactual changes caused by a change in the animals' state of arousal or movement. Threshold and I/O functions were repeatedly measured over the first 2 to 10 minutes. The stability and consistent course of recovery demonstrate that early threshold shifts are reproducible and that the slope of early I/O functions is not affected by the temporal order in which suprathreshold stimuli are presented.

Ongoing studies will assess cochlear changes, although given the short-term reversible nature of these physiologic changes, we expect that the EMLR-TTS is not associated with histopathology. This work confirms that moderately high levels of stimulation influence the responsiveness of higher centers of the auditory system to electric stimulation. These changes in both the threshold and amplitude of the EMLR waveforms are reversible. The level at which they occur is below the threshold for electric stimulation-induced histopathology. ${ }^{2.3,22,23}$ These changes occur at a charge density of less than $20 \mu \mathrm{C} / \mathrm{cm}^{2}$, well within the normal operating range of some prostheses. ${ }^{24}$ Local electrochemical changes at the electrode surface do not appear to be a likely component of the TTS, as impedance measured in several animals immediately following stimulation and in the presence of a TTS showed that they remain constant. Potential mechanisms underlying the EABR-TTS have been discussed by Miller et al. ${ }^{3}$ These considerations are equally relevant to the EMLR-TTS. It seems likely that metabolic factors contribute to this effect.

The use of this information to predict changes in the central auditory system could be of value in setting the safe upper limit of processor outputs in subjects who are difficult to test for cochlear implantation (eg, children and prelingually deafened adults). Intermediate experimental steps are required before this measure can be applied in humans, to relate changes in the EMLR to histologic change in the animal cochlea and to study the relationship of these EMLR-TTS to behavioral thresholds in animals. Finally, adult human subjects should be studied to assess shifts in behavioral and EMLR thresholds following moderately high levels of stimulation, which are within the limits of normal use. A systematic study of EABRTTS characteristics may be appropriate if a threshold shift is identified. The data from such a program of studies may lead to a new and reliable 

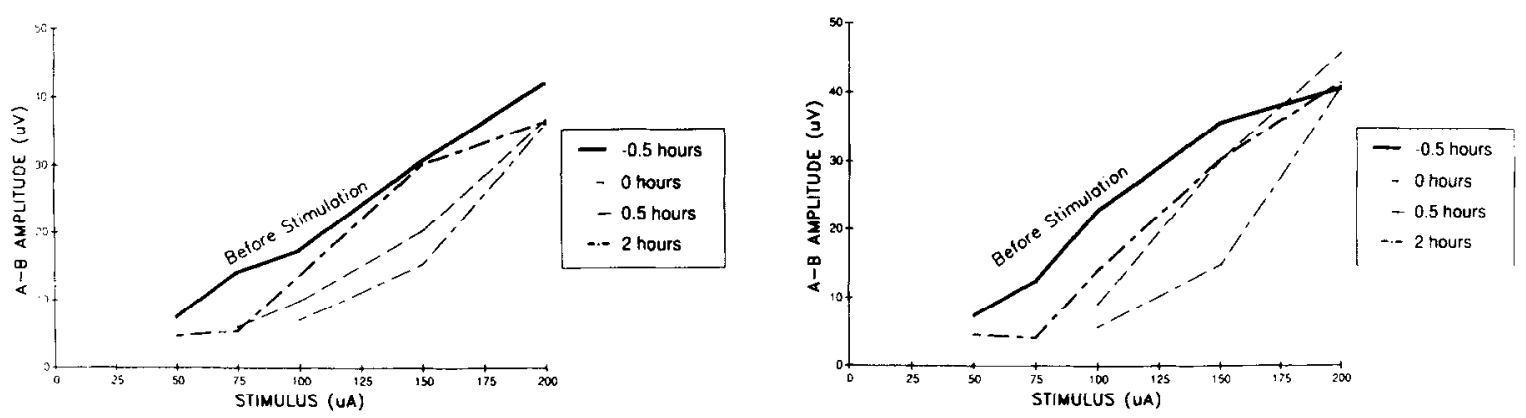

Figure 7. Representative $\mathrm{I} / \mathrm{O}$ functions before and after $200 \mu \mathrm{A}$ (left) and $300 \mu \mathrm{A}$ (right) sinusoidal stimulation.

measure for setting safe output limits for cochlear implant processors.

Acknowledgment. The authors would like to thank Yvonne Beerens for preparation of the manuscript.

\section{References}

1. Walsh SM, Leake-Jones PA: Chronic electrical stimulation of auditory nerve in cat: Physiological and histological results. Hearing Res 1982; 7:281-304

2. Miller JM, Duckert LG, Malone M, et al: Cochlear prosthesis: Stimulation-induced damage. Ann Otol Rhinol Laryngol 1983; 92:599-609

3. Miller JM, Duckert LG, Sutton D, et al: Animal models: Relevance to implant use in humans, in Schindler RA, Merzenich MM (eds): Cochlear Implants. New York, NY, Raven, 1985, pp 35-54

4. Game CJA, Gibson WPR, Pauka CK: Electrically evoked brain stem auditory potentials. Ann Otol Rhinol Laryngol 1987; 128:94-95

5. Allegra LA, Dobie RA: Electrically evoked ABR in the guinea pig following drug-induced deafness. Abstracts of the Fifth Midwinter Meeting of the Association for Research in Otolaryngology, 1982, p 4

6. Stypulkowski PH, van den Honert C: Characteristics of electrically evoked ABRs: I. Recordings from normal and pathological animals. Abstracts of the 1984 West Coast Cochlear Prosthesis Workshop, Battelle Institute, Seattle, WA, 1984

7. Smith L, Simmons FB: Estimating eighth nerve survival by electrical stimulation. Ann Otol Rhinol Laryngol 1983; 92:19-23

8. Lusted HS, Shelton C, Simmons FB: Comparison of electrode sites in electrical stimulation of the cochlea. Laryngoscope 1984; 94:878-882

9. van den Honert C, Stypulkowski PH: Characterization of the electrically evoked auditory brain stem response (ABR) in cats and humans. Hearing Res 1986; 21:109-126

10. Miyamoto RT: Electrically evoked potentials in cochlear implant subjects. Laryngoscope 1986; 96:178-185

11. Kileny PR, Kemink JL: Electrical evoked middle latency auditory potentials in cochlear implant candidates. Arch Otolaryngul Head Neck Surg 1987; 113:1072-1077

12. Musiek FE, Guerkink NA: Auditory brain stem and middle latency evoked response sensitivity near threshold. Ann Otol Rhinol Laryngol 1981; 90:236-240

13. Miller JM, Kileny PR, Burton M, et al: A cross-species comparison of acoustic and electric MLRs. Abstracts for the 12th Midwinter Meeting of the Association for Research in Otolaryngology, St Petersburg, FL, 1989, p 31

14. McGee TJ, Özdamar O, Kraus N: Auditory middle latency responses in the guinea pig. Am J Otolaryngol 1983; 4:116-122

15. Kraus N, Smith DI, Grossman J: Cortical mapping of the auditory middle latency response in the unanesthetized guinea pig. Electroencephalogr Clin Neurophysiol 1985; 62:219-226

16. Burton MJ, Miller JM, Kileny PR: Middle latency responses in guinea pigs: I. Electrical and acoustic stimulation. Arch Otolaryngol Head Neck Surg 1989; 115:59-62

17. Burton M, Miller JM, Kileny PR: Middle latency responses: II. Variation between stimulation sites. Arch Otolaryngol Head Neck Surg 1989; 115:458-461

18. Cohen MM: Coronal topography of the middle latency auditory evoked potentials (MLAEP) in man. Electroencephalogr Clin Neurophysiol 1982; 53:231-236

19. Wood CC, Wolpaw JK: Scalp distribution of human auditory evoked potentials. II. Evidence for overlapping sources and involvement of the auditory cortex. Electroencephalogr Clin Neurophysiol 1982; 54:25-38

20. Jyung RW, Miller JM, Cannon SC: Evaluation of eighth nerve integrity using the electrically evoked middle latency response. Otolaryngol Head Neck Surg 1989; 101:670-682

21. Crowther JA, Cannon SC, Miller JM, et al: Anesthesia effects on the electrically evoked middle latency response in guinea pigs. Am J Otolaryngol Head Ne.k Surg 1989; 101:51-55

22. Duckert LG, Miller JM: Acute morphological changes in guinea pig cochlea following electrical stimulation: A preliminary scanning electron microscopy study. Ann Otol Rhinol Laryngol 1982; 91:33-40

23. Leake-Jones PA, Rebscher SJ: Cochlear pathology with chronically implanted scala tympani electrodes. Ann NY Acad Sci 1983; 405:203-223

24. Shepherd RK, Clark GM, Black RC: Chronic electrical stimulation of the auditory nerve in cats. Acta Otolaryngol (Stockh) 1983; 399:19-31 (suppl) 